\title{
Stem cells from adipose tissue and breast cancer: hype, risks and hope
}

\author{
F Bertolini ${ }^{*}, 1$, J-Y Petit ${ }^{2}$ and M G Kolonin ${ }^{3}$ \\ ${ }^{1}$ Laboratory of Hematology-Oncology, European Institute of Oncology, Via Ripamonti 435, 20141, Milan, Italy; ${ }^{2}$ Division of Plastic \\ Surgery, European Institute of Oncology, 20141 Milan, Italy and ${ }^{3}$ Institute of Molecular Medicine, University of Texas Health \\ Science Center at Houston, Houston, TX, 77030, USA
}

Several recent papers have generated new hope about the use of white adipose tissue (WAT)-derived progenitor cells for soft tissue reconstruction in a variety of diseases including breast cancer $(\mathrm{BC})$, a procedure that is increasingly used worldwide. We revised the available literature about WAT cells and BC. In the BC field, we believe that the hype for the exciting results in terms of WAT progenitor cell engraftment and tissue augmentation should be tempered when considering the recent and abundant preclinical studies, indicating that WAT progenitors may promote BC growth and metastasis. White adipose tissue progenitors can contribute to tumour vessels, pericytes and adipocytes, and were found to stimulate local and metastatic BC progression in several murine models. Moreover, there are clinical retrospective data showing a significant increase in the local recurrence frequency in patients with intraepithelial neoplasia who received a lipofilling procedure for breast reconstruction compared with controls. Retrospective and prospective clinical trials are warranted to investigate in depth the safety of this procedure in BC. Preclinical models should be used to find mechanisms able to inhibit the tumour-promoting activity of WAT progenitors while sparing their tissue reconstruction potential.

White adipose tissue (WAT)-derived progenitor cells are increasingly used worldwide for soft tissue reconstruction in a variety of diseases including breast cancer, and encouraging cosmetic and functional results have been reported also in a randomised trial (Trojahn Kolle et al, 2013). However, recent and abundant preclinical studies suggest that WAT progenitors may promote breast cancer local growth and metastasis. Here, we briefly discuss the surgical use of WAT progenitors in breast cancer, the preclinical data about adipose tissue and WAT progenitor contribution to cancer progression and some possible future therapeutic interventions in this area.

\section{WAT PROGENITORS FOR BREAST RECONSTRUCTION AFTER SURGERY}

Breast cancer treatment is currently evaluated on cure rate figures and quality of life, and collaboration between breast and plastic surgeons has helped to decrease the disabling effect of surgical mutilation. Among the plastic surgery techniques currently available to reconstruct the breast, the lipofilling procedure is gaining a major interest. In fact, it is particularly efficient for reshaping the breast after total or partial reconstruction of this organ. The lipofilling technique consists in the localised injection of WAT-derived cells into the area of breast requiring a volume improvement after total breast reconstruction or after a local quadrantectomy or lumpectomy for a conservative treatment. The WAT-derived cells are collected by liposuction from different areas of the body; in most cases, liposuction is carried out on the abdomen or the thigh, and processed by centrifugation (see for a review, Bertolini et al, 2012). New techniques have been proposed to increase the concentration of WAT progenitor cells by means of an enzymatic treatment of the WAT collection, and several automated devices are currently being tested to enrich the lipofilling suspension for WAT progenitors before injection into the area of the breast to be refilled. In around $30-40 \%$ of cases, a reabsorption of the graft is observed; however, according to some authors, this and other similar side effects might be minimised with procedures aimed at increasing the fraction of high-density WAT cell suspensions (Amar et al, 2008).

\section{THE ROLE OF WAT CELLS IN CANCER LOCAL AND} METASTATIC CANCER

Cancer progression relies on stromal cells, a mixed population of fibroblastoid cells of both mesenchymal and haematopoietic

*Correspondence: Dr F Bertolini; E-mail: francesco.bertolini@ieo.it

Received 1 July 2014; revised 21 October 2014; accepted 3 December 2014; published online 13 January 2015 
origins shaping the tumour microenvironment (Kolonin et al, 2012). Collectively, these cancer-associated fibroblasts (CAF) (Karnoub et al, 2007) deposit extracellular matrix responsible for desmoplasia, stimulate vascularisation and epithelial-mesenchymal transition, as well as mute the antitumour immune response. These effects are executed by angiogenic, immunosuppressive, antiapoptotic and mitogenic factors secreted by CAF (Bertolini et al, 2012). While bone marrow-derived leucocytes functionally contribute to the tumour microenvironment, mesenchymal stromal cells (MSCs) appear to be the predominant progenitors of CAF (Karnoub et al, 2007). Mesenchymal stromal cells sense cancer as a site of hypoxia/ inflammation, which explains their trafficking to tumours (Kolonin et al, 2012). The trophic tumour milieu is composed of the same cell populations as the stroma/vasculature of WAT that surrounds cancers that are associated with obesity. These observations have led to our original hypothesis that WAT, overgrown in obesity, is a contributor to the pool of tumour stromal cells (Zhang et al, 2010). Low numbers of MSC are found in the bone marrow, whereas WAT contains large numbers of MSCs. Proliferation of these mesenchymal adipocyte progenitors, termed adipose stromal cells (ASCs), accompanies WAT expansion in obesity and a number of ASCs per gram of WAT increases in obese individuals. Adipose stromal cells contribute to the production of adipokines, the WATderived hormones, cytokines and growth factors. In this context, adipokines have been proposed to account for the link between obesity, manifested as WAT overgrowth, and breast cancer, as well as other cancers (Park et al, 2011). Although adipocytes, the lipid-storing WAT cells, are mainly active as endocrine adipokine producers, it is possible that ASCs, as well as monocytes/ macrophages, and other WAT-infiltrating leucocytes, may function as disseminators of paracrine adipokines. Systemic mobilisation of ASCs has been reported in obesity and is further elevated in cancer patients (Bellows et al, 2011a, b), suggesting the possibility of their trafficking to tumours (Figure 1).

There is no evidence that ASCs, or MSCs in general, are directly tumorigenic, although liposarcomas contain an aggressive multipotent malignant population resembling ASCs (Zhang et al, 2013). However, a body of data indicates that these cells may acquire a tumour-supporting function. By using mouse models, we have accumulated the original evidence that WAT promotes cancer progression by serving as a source of migratory cells secreting trophic factors from within the tumour (Zhang et al, 2009). Specifically, we have shown that ASCs can be recruited into tumours (Zhang et al, 2009) where they incorporate into blood vessels as pericytes and differentiate into adipocytes in an obesity-dependent manner (Zhang et al, 2012). These WAT-derived cells potentiate the supportive properties of the tumour microenvironment as witnessed by increased tumour vascularisation, as well as survival and proliferation of neighbouring malignant cells. In animal studies, administered ASC engraft tumours, which results in accelerated cancer progression (Zhang et al, 2009; Kidd et al, 2012; MartinPadura et al, 2012). Subsequent studies (Zhang et al, 2009, 2012; Martin-Padura et al, 2012; Orecchioni et al, 2013) suggest that increased vascularisation and adipogenesis promoted by ASC supports survival and proliferation of neighbouring malignant cells. Recent findings by us and others also suggest the role of WAT-derived stroma in cancer metastasis (Martin-Padura et al, 2012; Nowicka et al, 2013; Orecchioni et al, 2013; Rowan et al, 2014).

\section{LIPOFILLING AND BREAST CANCER RELAPSE: IS IT SAFE?}

The emerging data on the role of WAT cells in several types of neoplastic lesions has raised a question regarding the safety of mesenchymal stem cell therapies in cancer patients (Krumboeck et al, 2013). Provided the considerations above, this issue is particularly relevant to lipofilling in relation to breast reconstruction, where it is mandatory to rule out that the transfer of WAT cells into the breast excisional area might have detrimental effects to the patient. In fact, the possibility that breast cancer cells might still be present in the soft tissue after radical mastectomy or in the mammary parenchima after conservative treatment cannot be completely ruled out, and the injection of WAT cells in these areas might stimulate possibly 'dormant' cancer cells. It is therefore mandatory to demonstrate that the lipofilling procedure in breast cancer patients does not increase the risk of local or distant cancer recurrences.

The oncologic safety of lipofilling was focused as subject of investigation in 2009 by the American Society of Plastic Surgeons (ASPS) in a publication by the 'ASPS Fat Graft Task Force' (Gutowski and ASPS Fat Graft Task Force, 2009). Despite the fact that postlumpectomy and postmastectomy are clearly included in the indications for autologous WAT cell graft, the task force did not investigate or discuss adipocyte-stroma-cancer cell interactions in the context of development of local or metastatic recurrences. In the ASP task force project, we found only 6 out of 111 cited literature references that mentioned the potential impact of WAT cell autologous transfer on breast cancer detection, and none of these specifically investigated breast cancer incidence or recurrence. On the other hand, the task force also stated that based on a limited number of studies with few cases... no interference with breast cancer detection has been observed; however, more studies are needed...'.

A review of the literature published by Chan et al (2008) found only eight studies documenting clinical results. Only three had more than 50 patients and $>36$ months follow-up. Only two studies assessed the usefulness of fat grafting in reconstructive breast surgery. The discussion was focused only on complications of the technique and on the risk of microcalcifications occurring after fat transfer. No information was given on the local recurrence risk. A more recent systematic review by Krastev et al (2013) concluded that only 9 articles out of 269 mentioned an oncologic follow-up but only one with a match controlled group.

A match cohort study was performed at the European Institute of Oncology in Milan, Italy and gave rise to two different publications. In the first one, 321 consecutive patients operated for a primary breast cancer between 1997 and 2008 who subsequently underwent lipofilling for reconstructive purpose were compared with a matched control group of 642 patients with similar characteristics who did not undergo lipofilling and breast reconstruction with autologous WAT (Petit et al, 2012). Eightynine percent of the tumours were invasive. Median follow-up was 56 months from the primary surgery and 26 months from the lipofilling. Any event involving a local reappearance, such as local relapse or locoregional relapse or local relapse with synchronous metastases, was counted as local event (LE). Any other type of event was counted as a competing event. Eight and 19 patients had an LE in the lipofilling and control group, respectively, leading to comparable cumulative incidence curves $(P=0.792$; hazard ratio for lipofilling $v s$ no lipofilling $=1.11$ ( $95 \%$ confidence interval $0.47-2.64)$ ). These results were confirmed when patients undergoing quadrantectomy and mastectomy were analyzed separately and when the analysis was limited to invasive tumours. Based on 37 cases, the lipofilling group resulted at higher risk of LEs when the analysis was limited to intraepithelial neoplasia. In light of these observations, the same group published another paper focusing specifically on intraepithelial neoplasia, investigating 59 patients who had undergone lipofilling, with no recurrence between primary surgery and lipofilling (Petit et al, 2013). A control group included 118 matched patients with the corresponding recurrence-free intervals. Both groups were also matched for main breast cancer-related criteria. An LE was the primary end point, with follow-up starting from the baseline. 

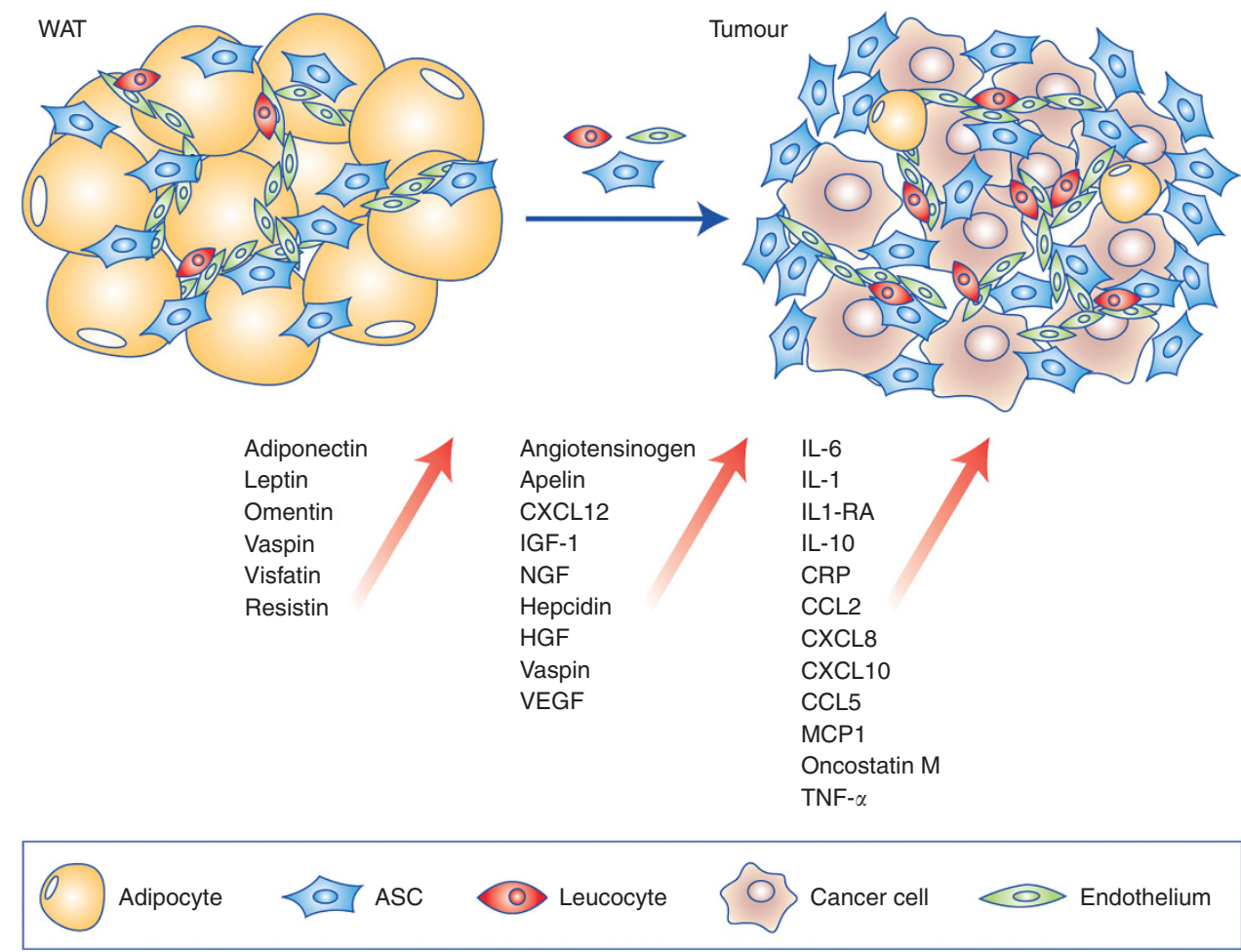

Figure 1. White adipose tissue is a source of ASCs recruited by tumours in obese patients. Secretion of indicated adipokines by these cells is proposed to explain the link between obesity and cancer. Abbreviations: CRP, C-reactive protein; CXCL, chemokine C-X-C motif ligand; HGF, hepatocyte growth factor; IL, interleukin; IGF-1, insulin-like growth factor-1; MCP1, monocyte chemotactic protein-1; NGF, nerve growth factor; TNF, tumour necrosis factor; VEGF, vascular endothelial growth factor.

Median follow-up was 63 and 66 months from surgery, and 38 and 42 months from baseline, for the lipofilling and control groups, respectively. The 5 -year cumulative incidence of LE was $18 \%$ and $3 \%(P=0.02)$. The LE was invasive in one case, invasive and in situ in three cases and pure in situ in five cases. The site of the LE was close to the lipofilling injection in $>90 \%$ of the cases.

The strength of these studies is the statistical methodology and the particular effort to match the control groups with the study groups. The difficulty was to match the groups according to the lap times without recurrence or metastasis knowing that the lap times was different for every patient. The weakness of the studies consists in the small population size and the short follow-up. Although these results should be confirmed in a larger series of patients and a longer follow-up is warranted, these data suggest that this topic deserves further in-depth clinical observation.

\section{CONCLUSIONS: UNMET NEEDS AND OPPORTUNITIES} FOR INTERVENTION

In the current dilemma between proposing to patients an interesting clinical intervention to improve their quality of life and the emerging evidence that WAT cells used for autologous breast reconstruction might have cooperative roles in breast cancer progression, we believe some actions should be taken.

First, plastic surgeons and statisticians should collaborate intensively to investigate the already available retrospective clinical data to better understand the safety of this procedure. Longer follow-up of lipofilling-treated patients are now available for in-depth retrospective investigations, and patients who are considering the lipofilling option deserve to be always and adequately informed about the risk-benefit balance. Along this line, prospective clinical studies should monitor the risk of cancer recurrence in patients treated with lipofilling procedures.
Second, the scientific community should generate better orthotopic models to investigate what WAT cell population (if any) is able to regenerate the breast without supporting, in turn, exit from dormancy of incumbent breast cancer cells. Based on these data, cell suspensions used for autologous breast reconstruction should be enriched for harmless cells and possibly depleted for other progenitors that are now known to promote breast cancer local and metastatic growth (e.g. endothelial progenitors and/or ASCs, see Orecchioni et al, 2013). Third, therapeutic interventions might be investigated preclinically and validated in clinical trials to reduce the relapse risk in cancer patients undergoing autologous breast reconstruction with WAT cells. For instance, there is increasing evidence that obesity-related metabolic dysregulation leads to inflammation, a process that promotes cancer progression in many different ways (Hursting and Dunlap, 2012). Thus, prevention of obesity seems particularly relevant for women who are at risk of developing cancer and undergoing lipofilling for breast reconstruction (Sirin and Kolonin, 2013). An indirect validation of this concept roots in the emerging data indicating that a low carbohydrate diet prevents cancer initiation and slows tumour growth in several murine models of cancer (Ho et al, 2011).

The biguanide Metformin, a safe drug commonly used for type 2 diabetes, has shown preclinical and clinical activity against breast cancer (Gronich and Rennert, 2013) and deserves to be investigated for the prevention of cancer recurrence, possibly also in this setting. In fact, we have preclinical evidence of a direct effect of Metformin against the proliferation of WAT progenitors in addition to breast cancer cells (Orecchioni et al, 2014). A number of candidate strategies and drugs might be considered in this context. Bone morphogenetic protein signalling, angiogenic pathways relying on VEGF and angiopoietin receptors, as well as chemotactic gradients regulated through CXCR1 and chemerin, are among candidates to be targeted for blunting the supporting roles of WAT progenitors in cancer (Klopp et al, 2012; Orecchioni et al, 2013). Finally, there are 
novel candidate drugs able to target WAT cells that deserve to be investigated also for the prevention of cancer recurrence (Sirin and Kolonin, 2013). Development of pharmacological approaches is underway. Of much promise are peptides binding to receptors selectively expressed on the surface of adipose cells. Such cellhoming peptides can be used as delivery vehicles in therapy targeting applications. Hunter-killer peptides, composed of a cell surface receptor-binding domain and a proapoptotic domain, have been developed and used for targeted cell ablation. A peptide targeting WAT endothelial cells through binding to cell surface prohibitin (Kolonin et al, 2004) is being tested clinically. Recently, an ASC surface marker, $\triangle \mathrm{DCN}$, has been identified, and a hunter-killer peptide targeting ASC through $\triangle \mathrm{DCN}$ has been shown to prevent renewal of white adipocytes in mice (Daquinag et al, 2014). Future studies will be needed to test the potential of compounds targeting WAT stromal/vascular cells, or adipocytes directly, to complement conventional cancer therapies.

As also suggested by Barkholt et al (2013), we believe that there is an urgent need for retrospective and prospective clinical trials along with regulatory viewpoints to safeguard the risk of tumorigenicity in MSC-based therapies. Basic and translational researchers, plastic and breast cancer surgeons, oncologists and statisticians should collaborate together to this aim.

\section{ACKNOWLEDGEMENTS}

This work was supported in part by AIRC (Associazione Italiana per la Ricerca sul Cancro), Fondazione Umberto Veronesi, Ministero della Salute and a Grant CNE-119003 from the American Cancer Society. FB is a scholar of the US National Blood Foundation. We apologise to the many researchers and authors whose relevant work we could not reference because of limitations in the size of the manuscript and in the number of references.

\section{CONFLICT OF INTEREST}

The authors declare no conflict of interest.

\section{REFERENCES}

Amar O, Bruhant-Rodier C, Lehmann S, Bollecker V, Wilk A (2008) Fat tissue transplant: restoration of the mammary volume after conservative treatment of breast cancers, clinical and radiological considerations. Ann Chir Plast Esthet 53: 69-77.

Barkholt L, Flory E, Jekerle V, Lucas-Samuel S, Ahnert P, Bisset L, Buscher D, Fibbe W, Foussat A, Kwa M, Lantz O, Mačiulaitis R, Palomäki T, Schneider CK, Sensebé L, Tachdjian G, Tarte K, Tosca L, Salmikangas P (2013) Risk of tumorigenicity in mesenchymal stromal cell-based therapies - bridging scientific observations and regulatory viewpoints. Cytotherapy 15: 753-759.

Bellows CF, Zhang Y, Chen J, Frazier ML, Kolonin MG (2011a) Circulation of progenitor cells in obese and lean colorectal cancer patients. Cancer Epidemiol Biomarkers Prev 20: 2461-2468.

Bellows CF, Zhang Y, Simmons PJ, Khalsa AS, Kolonin MG (2011b) Influence of BMI on level of circulating progenitor cells. Obesity 19: 1722-1726.

Bertolini F, Lohsiriwat V, Petit JY, Kolonin MG (2012) Adipose tissue cells, lipotransfer and cancer: a challenge for scientists, oncologists and surgeons. Biochim Biophys Acta 1826: 209-214.

Chan CW, McCulley SJ, Macmillan RD (2008) Autologous fat transfer - a review of the literature with a focus on breast cancer surgery. J Plast Reconstruct Aesthet Surg 61: 1438-1448.

Daquinag AC, Tseng C, Salameh A, Zhang Y, Amaya-Manzanares F, Dadbin A, Florez F, Xu Y, Tong Q, Kolonin MG (2015) Depletion of white adipocyte progenitors induces beige adipocyte differentiation and suppresses obesity development. Cell Death Differ 22: 351-363.

Gronich N, Rennert G (2013) Beyond aspirin - cancer prevention with statins, metformin and bisphosphonates. Nat Rev Clin Oncol 10: 625-642.

Gutowski KA. ASPS Fat Graft Task Force (2009) Current applications and safety of autologous fat grafts: a report of the ASPS fat graft task force. Plast Reconstr Surg 124: 272-280.

Ho VW, Leung K, Hsu A, Luk B, Lai J, Shen SY, Minchinton AI, Waterhouse D, Bally MB, Lin W, Nelson BH, Sly LM, Krystal G (2011) A low carbohydrate, high protein diet slows tumor growth and prevents cancer initiation. Cancer Res 71: 4484-4493.

Hursting SD, Dunlap SM (2012) Obesity, metabolic dysregulation, and cancer: a growing concern and an inflammatory (and microenvironmental) issue. Ann NY Acad Sci 1271: 82-87.

Karnoub AE, Dash AB, Vo AP, Sullivan A, Brooks MW, Bell GW, Richardson AL, Polyak K, Tubo R, Weinberg RA (2007) Mesenchymal stem cells within tumour stroma promote breast cancer metastasis. Nature 449: 557-563.

Kidd S, Spaeth E, Watson K, Burks J, Lu H, Klopp A, Andreeff M, Marini FC (2012) Origins of the tumor microenvironment: quantitative assessment of adipose-derived and bone marrow-derived stroma. PLoS One 7: e30563.

Klopp AH, Zhang Y, Solley T, Amaya-Manzanares F, Marini F, Andreeff M, Debeb B, Woodward WA, Schmandt R, Broaddus RR, Lu KH, Kolonin MG (2012) Omental adipose tissue-derived stromal cells promote vascularization and growth of endometrial tumors. Clin Cancer Res 18(3): $771-782$.

Kolonin MG, Saha P,K, Chan L, Pasqualini R, Arap W (2004) Reversal of obesity by targeted ablation of adipose tissue. Nat Med 10: 625-632.

Kolonin MG, Evans KW, Mani SA, Gomer RH (2012) Alternative origins of stroma in normal organs and disease. Stem Cell Res 8: 312-323.

Krastev TK, Jonasse Y, Kon M (2013) Oncological safety of autologous lipoaspirate grafting in breast cancer patients: a systematic review. Ann Surg 20: 111-119.

Krumboeck A, Giovanoli P, Plock JA (2013) Fat grafting and stem cell enhanced fat grafting to the breast under oncological aspects - recommendations for patient selection. Breast 22: 579-584.

Martin-Padura I, Gregato G, Marighetti P, Mancuso P, Calleri A, Corsini C, Pruneri G, Manzotti M, Lohsiriwat V, Rietjens M, Petit JY, Bertolini F (2012) The white adipose tissue used in lipotransfer procedures is a rich reservoir of CD34+ progenitors able to promote cancer progression. Cancer Res 72: 325-334.

Nowicka A, Marini FC, Solley TN, Elizondo PB, Zhang Y, Sharp HJ, Broaddus R, Kolonin M, Mok SC, Thompson MS, Woodward WA, Lu K, Salimian B, Nagrath D, Klopp AH (2013) Human omental-derived adipose stem cells increase ovarian cancer proliferation, migration, and chemoresistance. PLoS One 8: e81859.

Orecchioni S, Gregato G, Martin-Padura I, Reggiani F, Braidotti P, Mancuso P, Calleri A, Quarna J, Marighetti P, Aldeni C, Pruneri G, Martella S, Manconi A, Petit JY, Rietjens M, Bertolini F (2013) Complementary populations of human adipose $\mathrm{CD} 34+$ progenitor cells promote growth, angiogenesis, and metastasis of breast cancer. Cancer Res 73: 5880-5891.

Orecchioni S, Reggiani F, Talarico G, Mancuso P, Calleri A, Gregato G, Labanca V, Noonan DM, Dallaglio K, Albini A, Bertolini F (2014) The biguanides metformin and phenformin inhibit angiogenesis, local and metastatic growth of breast cancer by targeting both neoplastic and microenvironment cells. Int J Cancer; e-pub ahead of print 6 September 2014; doi:10.1002/ijc.29193.

Park J, Euhus DM, Scherer PE (2011) Paracrine and endocrine effects of adipose tissue on cancer development and progression. Endocr Rev 32: $550-570$.

Petit JY, Botteri E, Lohsiriwat V, Rietjens M, De Lorenzi F, Garusi C, Rossetto F, Martella S, Manconi A, Bertolini F, Curigliano G, Veronesi P, Santillo B, Rotmensz N (2012) Locoregional recurrence risk after lipofilling in breast cancer patients. Ann Oncol 23: 582-588.

Petit JY, Rietjens M, Botteri E, Rotmensz N, Bertolini F, Curigliano G, Rey P, Garusi C, De Lorenzi F, Martella S, Manconi A, Barbieri B, Veronesi P, Intra M, Brambullo T, Gottardi A, Sommario M, Lomeo G, Iera M, Giovinazzo V, Lohsiriwat V (2013) Evaluation of fat grafting safety in patients with intraepithelial neoplasia: a matched-cohort study. Ann Oncol 24: 1479-1484.

Rowan BG, Gimble JM, Sheng M, Anbalagan M, Jones RK, Frazier TP, Asher M, Lacayo EA, Friedlander PL, Kutner R, Chiu ES (2014) Human adipose 
tissue-derived stromal/stem cells promote migration and early metastasis of triple negative breast cancer xenografts. PLoS One 9: e89595.

Sirin O, Kolonin MG (2013) Treatment of obesity as a potential complementary approach to cancer therapy. Drug Discov Today 18: 567-573.

Trojahn Kolle SF, Fischer-Nielsen A, Mathiasen AB, Elberg JJ, Oliveri RS, Glovinski PV, Kastrup J, Kirchhoff M, Rasmussen BS, Talman ML, Thomsen C, Dickmeiss E, Drzewiecki KT (2013) Enrichment of autologous fat grafts with ex vivo expanded adipose tissue-derived stem cells for graft survival: a randomised placebo-controlled trial. Lancet 382: 1113-2020.

Zhang Y, Bellows CF, Kolonin MG (2010) Adipose tissue-derived progenitor cells and cancer. World J Stem Cells 2: 103-113.

Zhang Y, Daquinag A, Traktuev DO, Amaya F, Simmons PJ, March KL, Pasqualini R, Arap W, Kolonin MG (2009) White adipose tissue cells are recruited by experimental tumors and promote cancer progression in mouse models. Cancer Res 69: 5259-5266.

Zhang Y, Daquinag AC, Amaya-Manzanares F, Sirin O, Tseng C, Kolonin MG (2012) Stromal progenitor cells from endogenous adipose tissue contribute to pericytes and adipocytes that populate the tumor microenvironment. Cancer Res 72: 5198-5208.

Zhang Y, Young ED, Bill C, Belousov R, Peng T, Lazar AJ, Pollock RE, Simmons PJ, Lev D, Kolonin MG (2013) Heterogeneity and immunophenotypic plasticity of malignant cells in human liposarcomas. Stem Cell Res 11: 772-781.

(c) (1) (2)(2) This work is licensed under the Creative Commons Attribution-NonCommercial-Share Alike 4.0 Unported License. To view a copy of this license, visit http://creativecommons. org/licenses/by-nc-sa/4.0/ 